\title{
The local character of perspective organization
}

\author{
M. W. von GRÜNAU, S. WIGGIN, and M. REED \\ Queen's University, Kingston, Ontario, Canada
}

\begin{abstract}
The nature of perceived perspective orientation of 3-D objects in space was examined for Necker cube-like ambiguous figures. Using adaptation paradigms, it was found that processes of perspective orientation have restricted spatial extents with estimated diameters of about 3 deg. It was possible to fatigue these processes selectively, but independence was found to be incomplete. It is concluded that perspective orientation is not one global process, but consists of multiple, locally defined processes, whose spatial characteristics seem to be determined at an early stage of visual processing, but are integrated more globally.
\end{abstract}

The representation of three-dimensional space by artists or by our visual system has been full of puzzles for many centuries. In most instances, our view of the world, and of objects within this world, has a clear perspective orientation. That is, the arrangement of 3-D objects in 3-D space with respect to the viewer is usually unambiguous and consistent. This, hówever, is not true in all cases. There exist many powerful illusions, such as "magnetic hills," in which perspective orientation is distorted so that downhill looks like uphill. Many artists have realized the precariousness of our perspective organization. Escher, for instance, has made an art of this ambiguous situation (see Locher, 1971). He provides many examples in which perspective organization changes from one part of the picture to another, yet the picture as a whole seems to provide a consistent and unambiguous perspective view (e.g., Belvedere, Relativity).

The Necker cube is an example of a class of stimuli that is referred to as ambiguous or reversible, in this case with respect to perspective orientation. When one views these figures, they seem to switch between two equally likely organizations, each characterized by a different perspective orientation with respect to the viewer. It is usually assumed that we can see only one alternative at a time, never both together. Theoretical accounts of this phenomenon have been based, on the one hand, on either cognitive hypothesis testing (Gregory, 1970) or perceptual problem solving processes (Rock, 1975) and, on the other hand, on satiation processes with reciprocal inhibition (Attneave, 1971; Köhler, 1960) or auto-inhibition

We thank Andrea Riendeau and Heidi Walk for assistance in running Experiment 4, Lorraine Adams for the art work, and Audrey Bailey for preparing the manuscript. The research was supported by an operating grant (U0057) from the Natural Sciences and Engineering Research Council of Canada to $M$. W. von Grünau, which we gratefully acknowledge. A preliminary report of some of the experiments was presented at the European Conference on Visual Perception, Leuven, 1982.
(Howard, 1961) between two neural structures. Implicitly or explicitly, these accounts assumed that the underlying processes were general and global rather than multiple and local. Recent observations by Long and Toppino (1981), however, point to the existence of a number of more localized processes that are somewhat independent of each other. They demonstrated that the two alternative perspective organizations can be seen simultaneously when two Necker cubes are viewed. That is, one of them might be pointing up and to the right, while at the same time the other might point down and to the left. Then, at other times, both will point the same way. We decided to investigate this phenomenon in greater detail and to measure it quantitatively. We also wanted to bring under experimental control the amount of time during which same or opposite orientations were perceived.

\section{GENERAL METHODS}

In the following series of experiments, observers were presented, either monocularly or dichoptically, with light outline figures on a dark background (contrast $=82 \%$ ). The stimuli were projected onto white screens in a darkened room (background luminance = $3.4 \mathrm{~cd} / \mathrm{m}^{2}$ ) which the observers viewed via $45-\mathrm{deg}$ mirrors. Timing was controlled by Gerbrandt shutters. An adjustable foreheadand chinrest aided in the proper placement of the observers' eyes at a distance of $57 \mathrm{~cm}$ from the screens. The observers indicated their responses by pressing a button and holding it for the duration of the particular percept (i.e., perspective orientation). These responses were recorded on a chart-recorder and later analyzed. All observers had normal or corrected-to-normal vision, as tested with the Bausch \& Lomb Orthorater.

\section{EXPERIMENT 1}

\section{Method}

Our original observations were made in a dichoptic viewing situation. Influenced by this arrangenent, the stimuli were presented so that one figure was seen by the right eye $6 \mathrm{deg}$ to the right of a vertical binocular fixation line and the other figure was seen by the left eye 6 deg to the left of the line. The stimuli consisted of Neckercube-like figures (see in Figure 1 and Petersik, 1979) subtending 7.4 $\times 7.4 \mathrm{deg}$. 
Ten naive observers were tested to determine the proportion of viewing time during which the two alternative perspective orientations were seen simultaneously. Viewing periods were $15 \mathrm{sec}$ long, and each observer was tested three times. Prior to testing, all observers were familiarized with reversible Necker cubes and practiced on reporting the cubes' orientations (same or different).

\section{Results and Discussion}

The proportion of "different" responses varied greatly between observers. The average proportion was $33 \%$, with a range of $13 \%$ to $51 \%$ (see Figure 1 ). All observers reported the simultaneous presence of both orientations for a substantial part of the viewing period. Only "different" responses were reported here. Since the alternative category would not only include "same" percepts, but also "can't tell," etc., the obtained proportions underestimate slightly the actual percentage of time for which different, as opposed to same, orientations were perceived.

\section{EXPERIMENT 2}

In this experiment we attempted to manipulate the amount of time for which the two alternative organizations were seen simultaneously. For this we used an adaptation paradigm, in which observers were adapted by viewing unambiguous versions of the Neckercube-like figure and then tested with the ambiguous figures.

\section{Pilot Experiment}

In a pilot experiment, it was first established that adaptation effects did occur in the situation described. After a 30-sec pretest, during which observers indicated the proportion of time they saw orientation A (e.g., pointing left and down) when viewing the ambiguous figure, the observers viewed an unambiguous version (emphasizing either orientation A or orientation B) for $\mathbf{2}$ min. This was followed

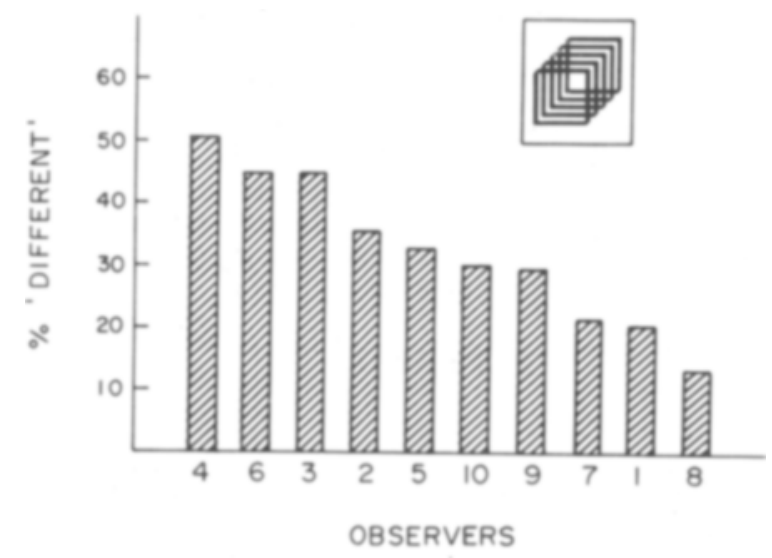

Figure 1. For each of 10 observers, the proportion of "different" reports is plotted in descending order. The inset shows the ambiguous test figure. The observers' reports relate to the situation when two such figures were seen In different perspective orientations simuitaneously. by a 30 -sec test (ambiguous figure) and two more sequences of 30-sec-readaptation and 30-sec-test periods. The adaptation figures are shown in the inset of Figure 2. During adaptation and test, the observers were free to move their eyes along the vertical fixation line to avoid fading. Exposure was monocular, and the stimuli had the same size and position as in Experiment 1.

Two experienced observers were tested in the pilot study, and their averaged results are presented in Figure $2 \mathrm{~A}$. The proportion of time orientation $\mathrm{A}$ was reported decreased by $57 \%$ after adaptation to orientation $A$ and increased by $33 \%$ after adaptation to orientation B. A statistical analysis showed these effects to be significant $[F(3,3)=18.2, p<.025]$. These results confirmed that adaptation is a powerful tool that can be used to manipulate perspective orientation of reversible figures. Similar effects have been obtained before (Carlson, 1953; Harris, 1979, 1980; Hochberg, 1950; Virsu, 1975), but the effect had to be established for the present situation and stimuli.

\section{Method}

The pilot study results were used in the main part of Experiment 2. As in Experiment 1, two ambiguous figures were presented dichoptically during the 30 -sec test period before and after the adaptation periods ( $120 \mathrm{sec} ; 2 \times 30$-sec readaptation). During adaptation, observers viewed two unambiguous versions which had either the same or different perspective orientations. During the tests, the observers indicated the proportion of time during which they saw both alternative orientations simultaneously (as in Experiment 1). To do so, they pressed a button every time and for as long as they perceived one ambiguous figure pointing left and down and simultaneously the other pointing right and up. When both figures were seen to point either left and down or right and up, they were to release the button. One naive and two experienced observers participated.

We made the following predictions: After the adaptation period, each test stimulus would be biased to appear for a longer time in the orientation opposite to the one experienced during adaptation. Therefore, when the adaptation stimuli consisted of two unambiguous figures pointing in the same orientation (either left and down or right and up), the proportion of time that both orientations were seen simultaneously would decrease. And when they consisted of two figures pointing in opposite orientations, the same proportion of time would increase. This followed from the results of the pilot study, when the two processes involved were assumed to be independent.

\section{Results and Discussion}

The results were similar for all three observers, and their averages are presented in Figure 2B. When adaptation occurred to two different perspective orientations, the two ambiguous figures were seen for a much larger proportion of time to be in opposite orientations (an increase of $75 \%$, from $30 \%$ to $53 \%$ ). Conversely, when adaptation was to the same orientations, the two ambiguous figures appeared in opposite orientations for a much smaller proportion of time (a decrease of $55 \%$, from $39 \%$ to $18 \%$ ). A statistical analysis suggests that these adaptation effects were highly significant $[F(1,2)=62.7, p<.013]$. 


\section{EXPERIMENT 3}

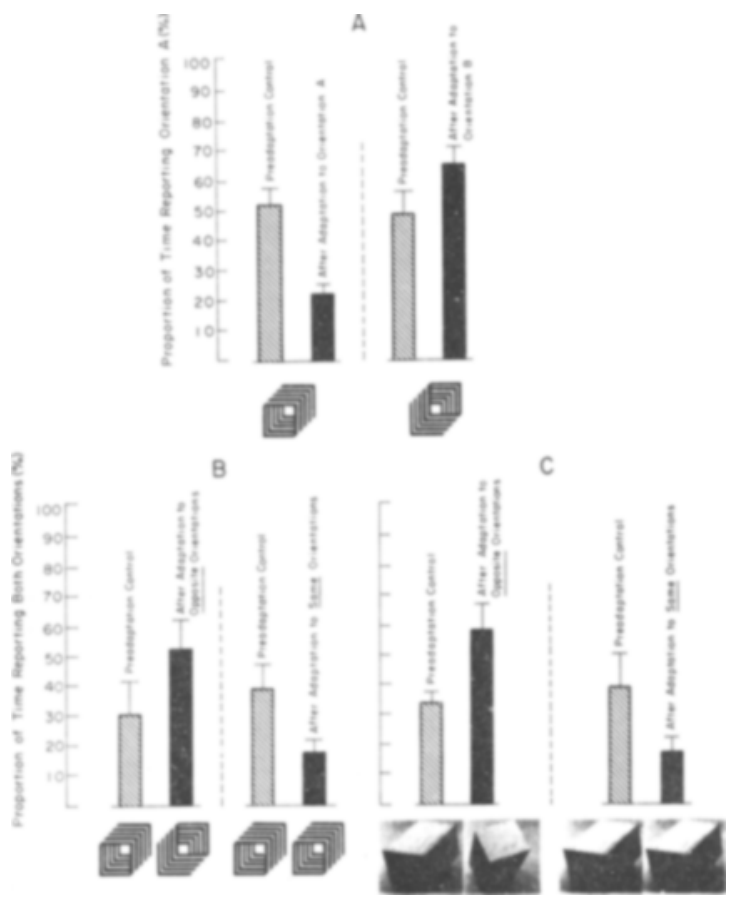

Figure 2. (A) Pilot study: The proportion of time for which the ambiguous test stimulus was percelved in orientation $A$ (left down) before and after adaptation to an unambiguous orientation $A$ and an unambiguous orientation $B$. The adaptation stimuli are shown below, along the $x$-axis. (B) The proportion of time for which both orientations were perceived simultaneously before and after adaptation to two unambiguous stimull in opposite or same orientations. Pairs of adaptation stimuli are shown below, along the $x$ axis. (C) Same as B, but for the pairs of photographic adaptation stimuli shown below, along the $x$-axis.

The same observers were also tested with a different pair of adaptation stimuli (see inset in Figure 2C). These were black/white photographs of an actual wooden box, positioned and illuminated in such a way as to emphasize the two perspective orientations used previously. The test stimulus remained the same ambiguous outline drawing of a Necker-cube-like figure. Except for the adaptation stimuli, the experiment was the same as before. The results are shown in Figure $2 \mathrm{C}$ as the average for the three observers. They were in all aspects very similar to the ones obtained for the outline drawings. Again, it was possible to manipulate the orientation of perspective organization separately for the two figures.

It is remarkable that the same adaptation effects occurred with stimuli that were in many basic aspects not similar to the test stimuli, that is, in contrast, orientation, and presence of contours. The drawn and the photographed figures, however, shared in common the same unambiguous perspectives of the original reversible figure. It is suggested that adaptation occurred within processes that were specifically tuned to perspective orientation.
In this experiment, the adaptation paradigm was used to assess the extent and localization of the proposed perspective processes.

\section{Method}

The observers were adapted to an unambiguous version (pointing left and down) of the usual ambiguous test figure. It was presented to the left eye at a visual direction of $2.5 \mathrm{deg}$ to the left of the fixation cross. Test stimuli consisted of the usual ambiguous figure (see Figure 1) and were presented either to the left eye or to the right eye. In either case, they could be in the same visual direction as the adaptation stimulus (2.5 deg left, T1 and T4), or 5 deg left (T2 and T5), or $2.5 \mathrm{deg}$ to the right of fixation (T3 and T6). These conditions are shown schematically in Figure 3A. Figure 3B summarizes how the various test stimuli relate to the adaptation stimulus with respect to visual direction, eye, and hemisphere. All stimuli were $2 \times 2$ deg and were presented monocularly according to the procedure described for Experiment 2, except that there were four readaptation periods. Adaptation occurred initially for $120 \mathrm{sec}$, followed by four 30-sec readaptation periods. Each of the five test periods was $30 \mathrm{sec}$ long. All experimental conditions were compared with corresponding control conditions with adaptation to an equal-luminance homogeneous field. The six test stimuli were presented in random order to five naive observers with normal or corrected-to-normal vision. The amount of time within the 30 -sec period for which the ambiguous test stimulus was seen in the "left and down" perspective orientation was measured after adaptation to the homogeneous field and after the unambiguous adaptation stimulus in a counterbalanced order across the five observers. The difference $\Delta t$ between the two measures constituted the adaptation effect for each of the five test periods.

\section{Results and Discussion}

A statistical analysis on these data ( 5 observers, 5 trials, 6 test stimuli) suggested significant effects for trials $[F(4,16)=4.2, p<.01]$ and test stimuli $[F(5,20)=$ $3.9, \mathrm{p}<.01]$. The trial effect suggested an increase in the amount of adaptation from the first adaptation to the last readaptation trial. Post hoc analyses of paired comparisons indicated, indeed, that the trial

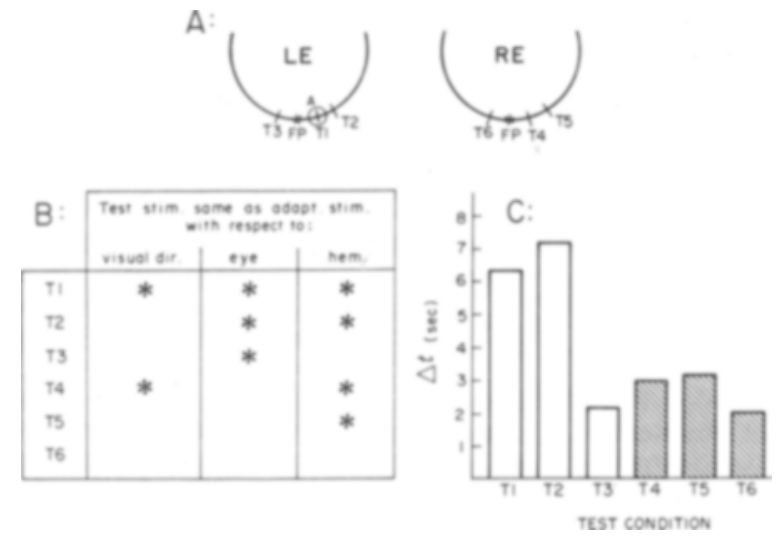

Figure 3. (A) Location of the adaptation (A) and test (T1 to T6) figures with respect to the fixation point (FP) on the retinas of the left eye (LE) and the right eye (RE). (B) Relationship between the location of the six test stimuli and the location of the adaptation stimulus with respect to visual direction, eye, and hemisphere. (C) Adaptation effect, expressed as the time difference $\Delta t(\sec )$ between seeing the left/down perspective after adaptation to left/ down and to a homogeneous field. Viewing time: 30 sec. 
effect was due to a difference between the first two and the last three adaptation measures. Since we were interested in strong adaptation effects for the analysis of the effects of the various test stimuli, further analyses were restricted to the last three adaptation measures. The respective means are shown in Figure $3 \mathrm{C}$ for all six test stimuli, as defined in Figure 3A. Paired comparison tests on these data indicated that test conditions $\mathrm{T} 1$ and $\mathrm{T} 2$ were both significantly different when tested against the other four conditions $[F(1,4)=7.4, p<.05 ; F(1,4)=7.3$, p < $.05]$, but were not different from each other $[F(1,4)$ $=.05, \mathrm{p}>.8 \mathrm{l}$. Comparisons among the other four conditions never reached significance. This permits the conclusion that large adaptation effects were observed only when the test stimulus was presented to the same eye and the same hemisphere as the adaptation stimulus, and when it appeared in the same visual direction or was $2.5 \mathrm{deg}$ off. That is, the assumed perspective process was restricted to a fairly small area in the retinal neighborhood of the position of the adaptation stimulus. The spatial extent of this process, however, was not tested in detail. It was not tested whether a greater separation between adaptation and test stimulus within the same half-retina would give similarly large adaptation effects.

\section{EXPERIMENT 4}

The extent of the restricted perspective process was tested in greater detail in this experiment. Again, an adaptation paradigm was used, similar to that of Experiment 3.

\section{Method}

Three observers (one experienced and two naive) were instructed to always fixate, and move their eyes along, a fixation circle (diameter: 12 min of arc) in the center of the display. Viewing was done with the left eye only. The ambiguous test figure (see Figure 1) was always presented in the center of the display before and immediately after each of the nine unambiguous adaptation figures (pointing to the left and down; the opposite perspective orientation was also examined in pilot experiments with corresponding results). Test and adaptation figures were $2 \times 2$ deg large. The nine adaptation conditions differed from each other by location of the stimuli along the vertical meridian with respect to the fixation circle. Thus, stimulus $A$ was presented at the position of the test stimulus. $A_{2}$ and $A_{6}$ were located .5 deg above or below the center, respectively. Similarly, $A_{3}$ and $A_{7}, A_{4}$ and $A_{6}$, and $A_{8}$ and $A_{9}$ were located $1,1.5$, and 2 deg, respectively, from the center of the fixation circle.

Each of the nine adaptation conditions was presented twice to each observer in different random orders. There was at least $1 / 2 h$ between different presentations. Each presentation began with the baseline measure, using the test stimulus. It was presented for $30 \mathrm{sec}$, and the observer indicated the time during which she/he perceived the ambiguous stimulus in the left/down orientation. Then the adaptation stimulus was presented for $2 \mathrm{~min}$, followed by a $30-\mathrm{sec}$ test period. Two readaptation periods of $30 \mathrm{sec}$, followed by 30 -sec test periods, completed this presentation. For each of the nine adaptation conditions, therefore, there were six measures from each observer.

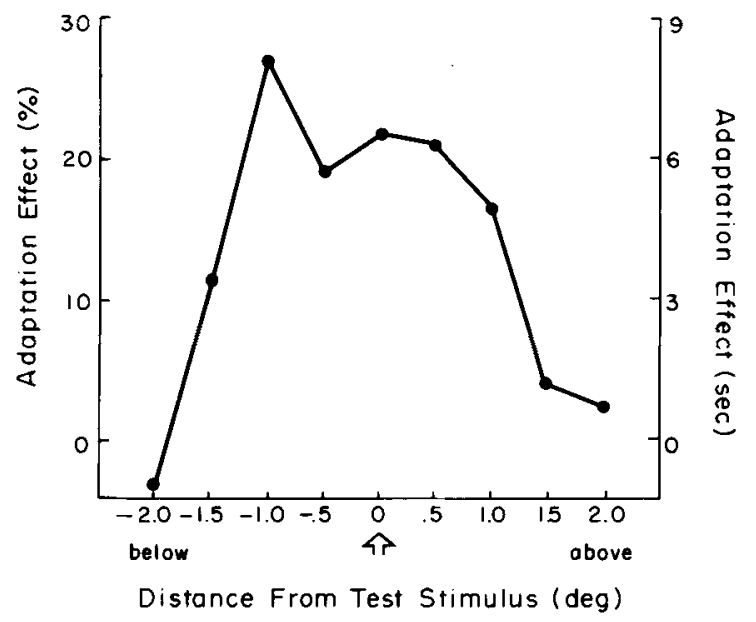

Figure 4. Adaptation effect as a function of the distance between the central test stimulus and the adaptation stimuli in degrees of visual angle.

\section{Results and Discussion}

For purposes of analysis, difference scores were calculated by subtracting each of the three postadaptation test scores from the preadaptation test score. Thus, the stronger the adaptation effect, the higher the positive difference score. Since initial analyses had indicated that there were no significant differences between the two presentations for each condition, these scores were combined for the main analysis of variance. This analysis showed that the three postadaptation scores were not different from each other $[F(2,4)=.44, p>.67]$, but that the nine adaptation conditions had significantly different effects $[F(8,16)=4.98, p<.004]$. These results are graphed in Figure 4 as the average for the three observers. Adaptation effects were strongest around the position of the test stimulus (arrow) and decayed with distance from that position in both directions. We have no explanation for the exceptionally large adaptation effect at the location $1.0 \mathrm{deg}$ below the test stimulus, but it occurred for all observers. From this outcome, it can be estimated that the adapted perspective process had a diameter of approximately 3 deg. This value is somewhat smaller than the one suggested by the previous experiment. It should be pointed out, however, that the position of the present process was central, whereas the previous one was $2.5 \mathrm{deg}$ in the periphery. The results, therefore, may indicate an increase in size of these perspective processes with increasing eccentricity.

\section{GENERAL DISCUSSION}

The main conclusions that we want to draw from the above experiments are the following: (1) Perspective orientation is not one global process, but consists 
of multiple processes that can be defined locally but are integrated more globally. (2) These localized processes have a restricted spatial extent, which can be estimated to have, in the center of the visual field, a diameter of about $3 \mathrm{deg}$, which probably increases with eccentricity. (3) These processes can be manipulated selectively, but are only partially independent. (4) The spatial characteristics of these processes seem to be determined at an early stage of visual processing (possibly retinal).

At the outset, it is important to establish that the processes we were studying were, indeed, those of perspective orientation. The results of Experiment 2, which were identical for drawn (outline) and photographed adaptation figures using the same outline test figure, seem to indicate quite convincingly that stimulus features, such as orientation and contrast of contours, specific angles, etc., were not responsible for the observed adaptation effects. Rather, the underlying processes seem to have been attending primarily to the perspective orientation of the figures, which was common to all. This conclusion is also supported by the fact that small eye movements along the fixation line, which were encouraged to prevent fading, did not prevent adaptation from occurring. At the same time, these movements would tend to prevent adaptation effects to local features in particular locations. One possible problem with the present results may be certain response biases of the observers or the demand characteristics of the given tasks. Petersen and Hochberg (1983) recently addressed this problem and provided a procedure (opposed-set method) to measure these influences. In the present case, we attempted to minimize these effects by making instructions as symmetrical as possible. In the adaptation experiments, opposite adaptation conditions were used to detect possible biases. The pattern of results indicates that these biases cannot explain the present effects.

One might then be tempted to follow a suggestion made by Long and Toppino (1981) and refer to these processes as "perspective channels." This definition specifies only their restricted spatial extent and, at this point, does not address their range of perspective orientations. The assumption of a multitude of restricted channels covering the visual field, presumably with much overlap, necessarily implies channel independence also, at least at an early stage. This kind of independence is suggested by our ability to selectively manipulate the activity in particular channels. It does not seem to be complete, however, since the alternative organizations of the ambiguous figure were perceived simultaneously only for an average of about $33 \%$ of the time, rather than for the predicted $50 \%$. This prediction is derived from the assumption that in the case of two figures we have four equally probable outcomes: both pointing up, both pointing down, one pointing up, the other down, and one pointing down, the other up. This assumption may not be correct, however. It has been known for some time that the alternative percepts in ambiguous figures often are not equally likely (e.g., Adams \& Haire, 1958; Carlson, 1953; Harris, 1980; Long, Toppino, \& Kostenbauder, 1983; Orbach, Ehrlich, \& Heath, 1963; Palmer \& Bucher, 1981; Price, 1969). The present results could then be predicted if one alternative had been seen for $80 \%$ of the time and the other for only $20 \%$. This bias would become even stronger when we had two figures. Then both would be pointing in one direction for $94 \%$ of the time and in the other for only $6 \%$. Even though the partial times (i.e., for both pointing down vs. both pointing up) were not determined, it is quite clear that no such bias was ever observed here. Some amount of "crosstalk," therefore, seems to occur between the channels, perhaps at a later stage, causing the preferred operation of channels selective for similar orientations of perspective organization.

In other words, the local channels can be influenced more globally by neighboring channels to permit the establishment of one overall perspective organization. This is certainly the case under normal, unrestricted conditions. Local ambiguities of perspective will be decided by the global perspective organization. In our restricted situations, however, we were able to reveal the local character of perspective organization.

Similar conclusions, suggesting various kinds of interactions between local and global processes in perceptual organization, have been reported recently by a number of authors using a variety of reversible figures (Gillam, 1972; Hochberg, 1981; Long et al., 1983; Palmer \& Bucher, 1981, 1982; Petersen \& Hochberg, 1983). The general picture that emerges, not only for perspective organization, is that early analyses are performed on local levels, but only some will be consistent with processes at more global levels. Thus, global levels will determine the selection at the local levels, perhaps according to Hebbian rules (Hebb, 1949).

\section{REFERENCES}

Adams, P. A., \& Haire, M. (1958). Structural and conceptual factors in the perception of double-cube figures. American Journal of Psychology, 71, 548-556.

AtTneave, F. (1971). Multistability in perception. Scientific American, 225(6), 62-71.

Carlson, V. R. (1953). Satiation in a reversible perspective figure. Journal of Experimental Psychology, 45, 442-448.

Gillam, B. (1972). Perceived common rotary motion of ambiguous stimuli as a criterion of perceptual grouping. Perception \& Psychophysics, 11, 99-101.

Gregory, R. L. (1970). The intelligent eye. New York: McGrawHill.

Harris, J. P. (1979). The Schröder staircase: A new perspective. Perception \& Psychophysics, 26, 312-318. 
Harmis, J. P. (1980). How does adaptation to disparity affect the perception of reversible figures? American Journal of Psychology, 93, 445-457.

HeB, D. O. (1949). Organization of behavior. New York: Wiley. HochBerg, J. E. (1950). Figure-ground reversal as a function of visual satiation. Journal of Experimental Psychology, 40, 682-686.

Hochberg, J. E. (1981). Levels of perceptual organization. In M. Kubovy \& J. Pomerantz (Eds.), Perceptual organization. Hillsdale, NJ: Erlbaum.

Howard, I. P. (1961). An investigation of a satiation process in reversible perspective of revolving skeletal shapes. Quarterly Journal of Experimental Psychology, 13, 19-33.

KöHLER, W. Dynamics in psychology. New York: Grave Press.

LOCHER, J. L. (1971). The world of M. C. Escher. New York: Abrams.

Long, G. M., \& Toppino, T. C. (1981). Multiple representations of the same reversible figure: Implications for cognitive decisional interpretations. Perception, 10, 231-234.

Long, G. M., Toppino, T. C., \& Kostenbauder, J. F. (1983). As the cube turns: Evidence for two processes in the perception of a dynamic reversible figure.

Orbach, J., Eirlich, D., \& Heath, H. A. (1963). Reversibility of the Necker cube: I. An examination of the concept of "satia- tion of orientation." Perceptual and Motor Skills, 17, 439-458.

Palmer, S. E., \& Bucher, N. M. (1981). Configural effects on perceived pointing of ambiguous triangles. Journal of Experimental Psychology: Human Perception and Performance, 7, 88-114.

Palmer, S. E., \& Bucher, N. M. (1982). Textural effects in perceived pointing of ambiguous triangles. Journal of Experimental Psychology: Human Perception and Performance, 8, 693-708.

Petersen, M. A., \& Hochenro, J. (1983). Opposed-set measurement procedure: A quantitative analysis of the role of local cues and intention in form perception. Journal of Experimental Psychology: Human Perception and Performance, 9, 183-193.

Petersik, J. T. (1979). Optical defocusing reverses perceptual organization. Perception, 8, 225-228.

Price, J. R. (1969). Effect of extended observation on reversible perspective duration. Psychonomic Science, 16, 75-76.

Rock, I. (1975). An introduction to perception. New York: Macmillan.

Virsu, V. (1975). Determination of perspective reversals. Nature, 257, 786-787.

(Manuscript received November 23, 1983; revision accepted for publication February 23, 1984.) 